\title{
Role of imaging in novel mitral technologies-echocardiography and computed tomography
}

\author{
Catalin Loghin, Andrei Loghin \\ Cardiology Division, UTHealth McGovern Medical School, Houston, TX, USA \\ Correspondence to: Catalin Loghin. Cardiology Division, Room MSB 1.246, 6431 Fannin, Houston, TX, USA. Email: catalin.loghin@uth.tmc.edu.
}

\begin{abstract}
As minimally invasive cardiovascular procedures gain popularity, novel transcatheter mitral valve repair devices continue to emerge. The success of these technologies is critically dependent on high quality imaging performed at all stages: patient selection, intervention planning, intraprocedural guidance, monitoring complications and follow-up. We present an overview of specific imaging requirements and challenges applicable to mitral valve interventional techniques. Modern valve imaging is multimodal and primarily combines echocardiography and computed tomography (CT). Echocardiography remains the gold standard for detailed anatomic imaging, complete hemodynamic characterization and real-time guidance and evaluation of procedural success. CT is indispensable for mitral annulus (MA) imaging and in predicting left ventricular outflow tract (LVOT) obstruction post transcutaneous mitral valve replacement (TMVR). 3D modeling, fusion imaging and automated image analysis may further contribute to the evolutionary transformation of valvular heart imaging.
\end{abstract}

Keywords: Structural heart disease; mitral technologies; interventional imaging; echocardiography; cardiac computed tomography

Submitted Jun 27, 2018. Accepted for publication Sep 20, 2018.

doi: 10.21037/acs.2018.09.07

View this article at: http://dx.doi.org/10.21037/acs.2018.09.07

\section{Introduction}

As the focus of structural heart disease is shifting toward new mitral valve technologies, imaging the complex mitral valve anatomy has become a challenging and rapidly evolving field. Real-time 3D echocardiography is now standard procedure, computed tomography (CT) has gained new indications in valve imaging and "interventional imaging" emerged as a new subspecialty.

\section{Mitral valve-functional anatomy}

Individual mitral valve apparatus components require specific imaging protocols and techniques.

(I) The mitral annulus (MA) is mathematically represented as a hyperbolic paraboloid, with a higher anterior peak included in the aorto-mitral curtain, a lower posterior peak and two fibrous trigones, representing its lateral and medial nadirs. The flat anterior annulus is the least mobile portion, in contrast to the rounded posterior aspect, which is more "contractile". Due to this dynamic shape, MA may be difficult to characterize. Defined as the leaflet insertion zone, the anterior annulus is cranial to the actual hinge point of the anterior mitral leaflet (AML), due to the fibrous substrate of the aorto-mitral curtain, which extends below leaflet insertion (Figure 1). In patients with myxomatous degeneration, the leaflet insertion may be displaced above the annulus, resulting in "mitral disjunction" (Figure 2, Video 1) (1). Distinguishing between the MA and AML hinge point may be relevant for transcatheter mitral valve (TMVR) procedures. MA geometry changes during the cardiac cycle. In systole, the annulus descends apically, becomes more vertical and increases its anteroposterior diameter. The saddle shape is accentuated, which reduces leaflet strain. In diastole, the annulus becomes more horizontal, shrinks its area and flattens. The aortomitral coupling determines the angle between aortic and mitral annuli, a critical parameter for 


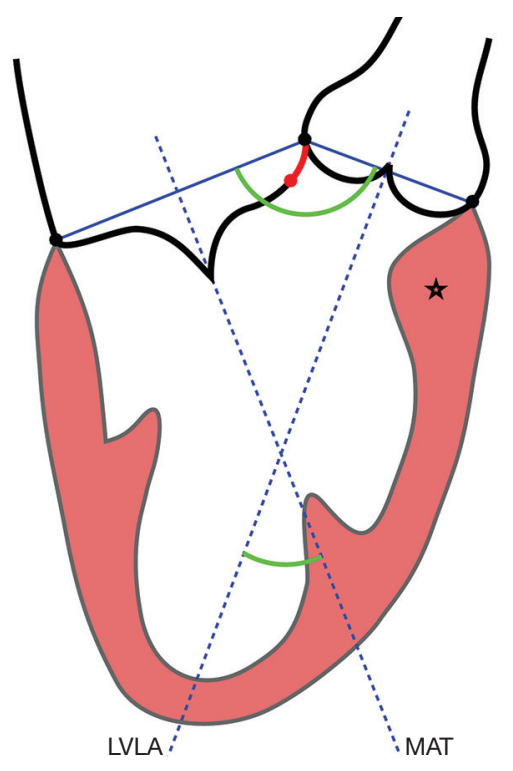

Figure 1 Note the angle between mitral annulus trajectory (MAT) and LV long axis (LVLA). Green arcs: aorto-mitral angle (AMA) and MAT-LVLA angle. Solid blue lines: mitral and aortic planes. Red line and dot: aorto-mitral curtain and AML hinge point, respectively. *, hypertrophy of the basal segment of the interventricular septum, potentially contributing to LVOT obstruction. predicting left ventricular outflow tract (LVOT) obstruction following TMVR. The mitral annulus "trajectory", defined as a vector orthogonal to the annulus "plane", commonly diverges from the left ventricular longitudinal axis and determines the site for transapical device delivery (Figure 1). Lastly, the posterior MA tends to be less compliant, resulting in asymmetric, typically anterior, deployment of TMVR devices. Locating the MA during a procedure may require visual aids: an angiogram of the left circumflex artery, mitral annulus calcification (MAC) noted on fluoroscopy, or a guidewire deployed in the coronary sinus.

(II) Mitral leaflets are quite different in structure, size, shape and strain exposure. Two commissures, anterolateral and posteromedial, separate the anterior "aortic" leaflet from the posterior "mural" leaflet and may not correspond to the trigones. The Carpentier nomenclature defines, for each leaflet, three scallops, separated by incomplete clefts, and establishes a common language between cardiologists and cardiovascular surgeons. Anatomic variants may be important for edge-to-edge repair procedures: absence of commissures and

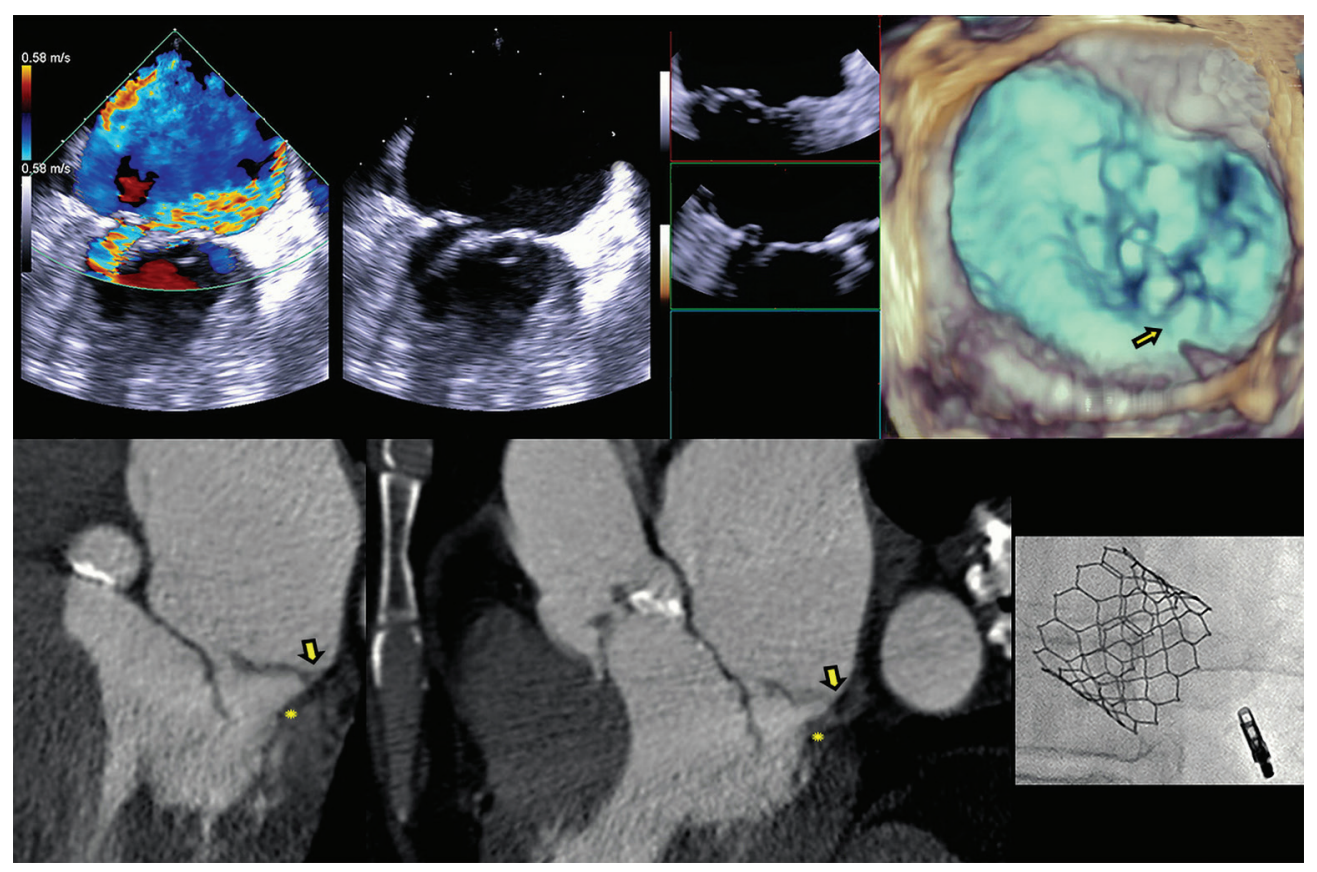

Figure 2 DMR: posterior mitral leaflet with flail central scallop (P2) flail. Disjunction noted on 3D TEE and CT, 30\% systolic phase. Severe aortic stenosis was present; patient underwent concomitant TAVR and MitraClip ${ }^{\circledR}$ valve repair. DMR, degenerative mitral regurgitation; TEE, transesophageal echocardiography; TAVR, transcatheter aortic valve replacement. *, mitral annulus location; Arrow: actual leaflet insertion. 
continuity between leaflets, or additional and deeper clefts, with potential valvular regurgitation. Scallops may become apparent in diastole only, while leaflet redundancy is easily noticed in systole. Structurally, the wider, shorter, fan shaped, anterior leaflet displays a fibrous continuity with the noncoronary and left coronary aortic valve cusps and is very mobile. In contrast, the narrower, longer, band shaped, posterior leaflet attaches to a rather discontinuous fibrous skeleton, which renders it prone to distension, and has limited excursion. Valve competency is a product of the complete coaptation of leaflet edges, which results in systolic tenting of the valve, with all scallops remaining under the mitral annulus "plane". Valve prolapse is defined as scallop systolic displacement of $\geq 3 \mathrm{~mm}$ above the MA, whereas flail is defined as a leaflet edge which has lost its coaptation and oscillates freely within the left atrium. Functionally, the posterior leaflet is exposed to an overall higher strain compared to its anterior counterpart. This explains the frequent involvement of the posterior leaflet in pathologic processes, such as annulus calcification, which occurs predominantly in the posterior annulus, or myxomatous degeneration, which commonly involves the central posterior scallop (2).

(III) Abnormalities of chordae tendineae and papillary muscles may impact any mitral procedure: false ventricular tendons, short chordae, direct leaflet insertion, or tethering and apical displacement of papillary muscles.

\section{Mitral valve imaging-multimodality approach}

To meet the needs of the large variety of mitral valve devices, multimodality imaging routinely combines echocardiography and computed tomography (3).

(I) Echocardiography, transthoracic (TTE) or transesophageal (TEE), is indispensable for diagnosis, procedure planning, intra-procedural guidance, monitoring complications and for patient follow-up. It is the imaging modality with the highest temporal resolution, adequate spatial resolution and the only one that can provide complete hemodynamic data. Several comprehensive reviews detail imaging protocols dedicated to mitral valve, as well as patient selection criteria for mitral procedures (3-5). Echocardiography can easily distinguish between degenerative and functional mitral regurgitation (DMR and FMR, respectively). DMR is a disease of the valve, which undergoes myxomatous degeneration, affecting the morphology and function of various structures: leaflet thickening, redundancy, prolapse and disjunction, or chordal rupture with a flail leaflet (Figure 2, Video 1) (6). DMR associates severe annulus dilatation, with a highly eccentric regurgitant jet that peaks at end systole and is oriented away from the diseased leaflet. In contrast, FMR is a disease of the ventricle, where a tethered or apically displaced papillary muscle impairs the closure of an otherwise morphologically normal valve, followed by annular dilatation. The FMR regurgitant jet peaks in early systole, is less eccentric and is oriented toward the culprit leaflet. Arguably, the severity criteria for FMR versus DMR may be different $(5,7)$. The regurgitant jet volume is influenced by intravascular volume and left ventricular afterload. This explains the significant changes in MR severity assessment between interval examinations. TEE tends to overestimate MR severity, compared to TTE, if only a semiquantitative assessment of color Doppler is used. MR evaluation should be performed only during euvolemic state, keeping the recommended Nyquist limits for color Doppler $(50-60 \mathrm{~cm} / \mathrm{sec})$ and proximal isovelocity surface area (PISA) measurements. Quantitative methods may overestimate highly eccentric or nonholosystolic jets. Gross errors commonly result from analysis of single frame color Doppler images, ignoring the dynamic changes of the regurgitant orifice area and jet volume. Lastly, the angiographic classification of MR severity does not overlap the echocardiographic severity grading. As none of the echocardiographic techniques fully characterizes $\mathrm{MR}$, the proper evaluation of this condition requires considerable skill and experience. Mitral stenosis (MS) severity is largely based on valve area, with a threshold of $1.5 \mathrm{~cm}^{2}$, as transmitral gradients are highly variable and related to the heart rate and transmitral flow. While such a simplification is appealing, relying on valve area only may lead to inappropriate clinical decisions. Area measurement is prone to technical errors, using only planimetry of $2 \mathrm{D}$ cross sectional views, $3 \mathrm{D}$ ventricular views of leaflet edges, or 3D atrial views of the funnel shaped, calcified valve, where clearly defined measurement 


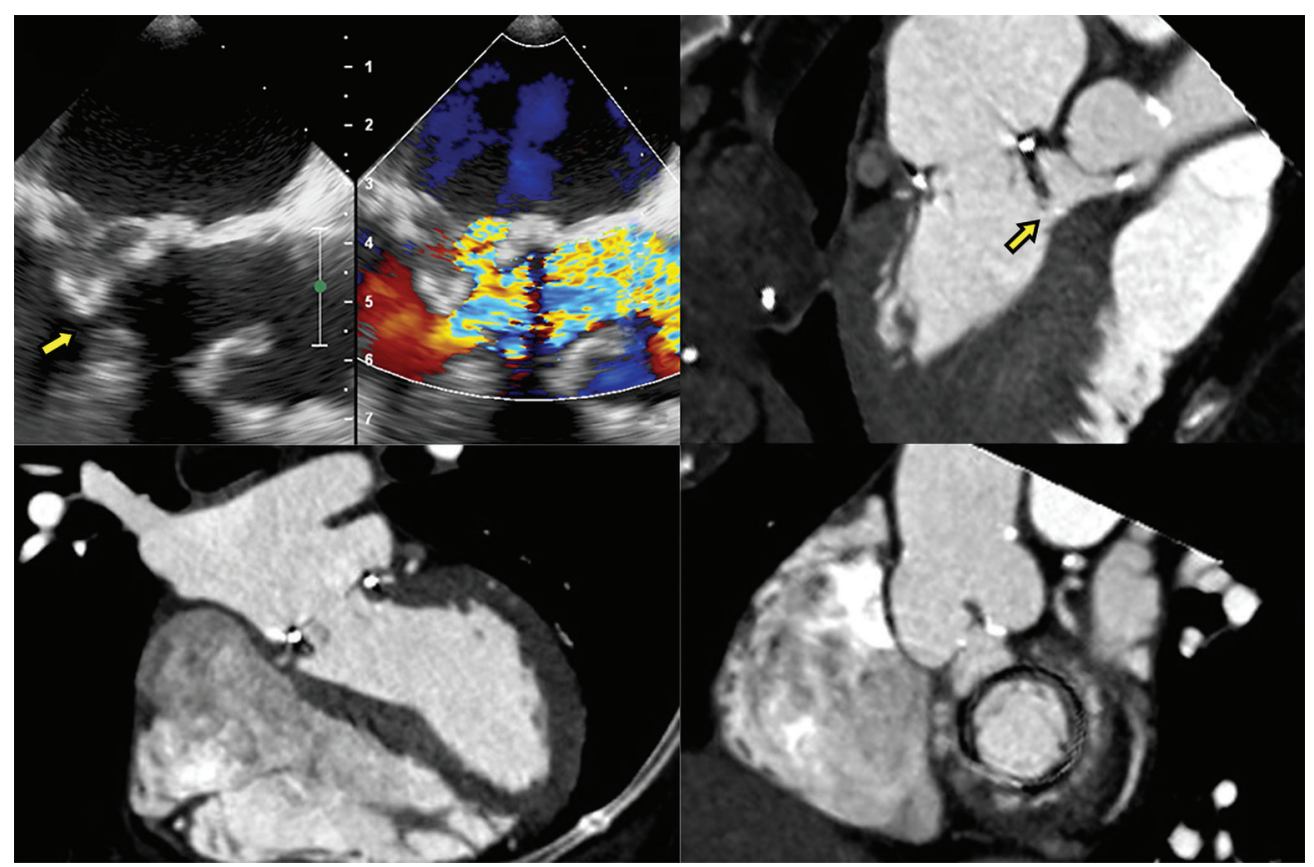

Figure 3 LVOT obstruction: severe systolic LVOT obstruction, between prosthesis strut and hypertrophied basal segment of the interventricular septum (arrows). Prosthesis with normal morphology and function. CT viewing parameters: kernel B26f, W 820, L 375, to improve prosthesis leaflet definition. LVOT, left ventricular outflow tract.

points are lacking. It is important to understand the difference between the geometric valve area resulting from planimetry and the smaller effective area of Doppler based methods: pressure half-time and continuity equation. In patients with symptoms out of proportion to TTE findings, supine bicycle exercise echocardiography reveals increased, exercise-induced mitral gradients and pulmonary systolic pressure, correlated with stress induced symptoms, even in the presence of a valve area $>1.5 \mathrm{~cm}^{2}$ (8). Definition of device success in mitral procedures relies on criteria published by the Mitral Valve Academic Research Consortium (MVARC): reduction of $M R$ to < moderate, transmitral gradient $<5 \mathrm{mmHg}$, effective valve area $>1.5 \mathrm{~cm}^{2}$, and LVOT gradient $<20 \mathrm{mmHg}$ (9). These parameters are measured 30 days post-procedure to allow for left atrial remodeling and functional recovery. One must again remember the difference between geometric and effective areas, which is more pronounced for the slit-like orifice of mitral stenosis, compared to the more rounded orifice of a TMVR device.

(II) CT, compared to echocardiography, lacks hemodynamic data, has lower signal to noise ratio, comparable temporal resolution and better spatial and contrast resolution, making it suitable for MV imaging (10). Operator-dependent, optimized imaging protocols for MV include: wide field of view, higher peak $\mathrm{kV}$, bolus timing using time attenuation curves, left atrial contrast target, $5-10 \%$ phase intervals and specific reconstructions for annulus sizing and LVOT analysis (11). Image analysis is performed on the thinnest possible slice, using projections similar to echocardiographic views, with 4D reconstructions and multiplanar reformatting adjusted to minimize beam hardening and blooming effect. Lifelike, 4DCT cinematic rendering may be more useful compared to traditional 3D volumetric reconstructions. CT is indispensable for mitral annulus sizing and for the prediction and evaluation of LVOT obstruction risk in TMVR (Figure 3, Video 2) (10,12-22). Additionally, CT allows precise measurement of the geometric mitral valve area, predicts optimal fluoroscopic angles and identifies the location of the coronary sinus in relation with the annulus (Figure 4). Accurate sizing of the MA is critical for device choice. The least square planes method projects the $3 \mathrm{D}$ contour of the 
annulus on to a 2D plane and led to the concept of a simplified, flat, D-shaped mitral annulus, which reflects the actual planar landing zone of TMVR devices $(6,23,24)$. A reproducible "effective annular diameter" can be derived from either the measured area, or the perimeter of the annulus (12). CT is vastly superior to echocardiography in imaging
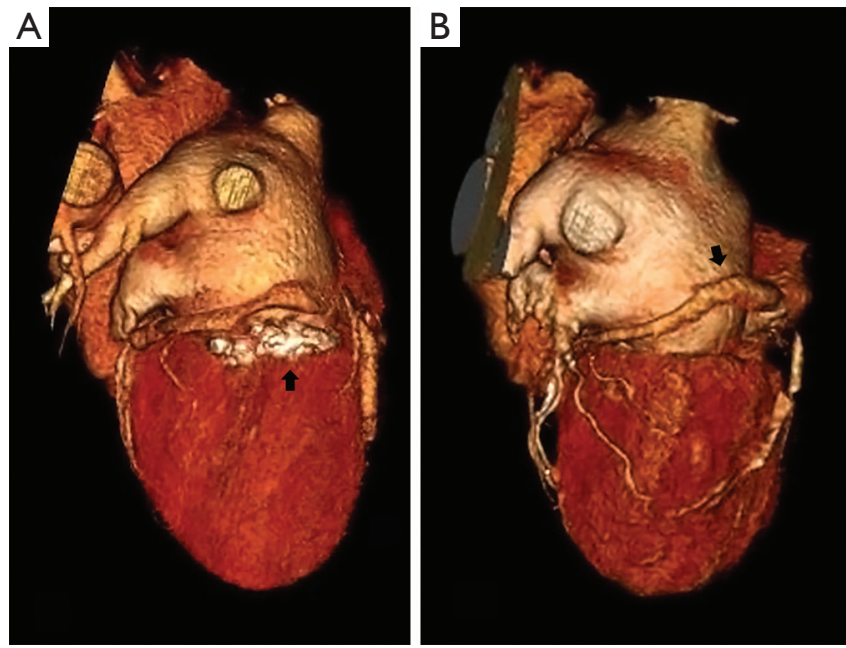

Figure 4 Coronary sinus and MAC-3DCT volumetric rendering. (A) MA location is accurately indicated by MAC (arrow), which displaces and distorts the coronary sinus; (B) coronary sinus (arrow) located well above MA. MA, mitral annulus; MAC, mitral annulus calcification. mitral annulus calcification (MAC), which can disrupt the atrioventricular groove and extend into the leaflets or the adjacent myocardium. Extensive leaflet calcification or fibrosis represents the basis of "degenerative MS" (Figure 5). MAC may involve the adjacent myocardium, or display a "caseous" core, without significant MS (Figure 6). TMVR creates a "neo-LVOT" between the basal segment of the interventricular septum and the AML, displaced anteriorly by the prosthesis struts, or flange (25). The semilunar cross-sectional area of the neo-LVOT may be measured by planimetry. Since LVOT obstruction may have profound hemodynamic consequences, several methods have been proposed to predict the risk of this complication, including proprietary software (11,24-26). Predictors of LVOT obstruction can be identified by CT, TTE or TEE: aortomitral angle $<110^{\circ}$, LVOT area $<2.0 \mathrm{~cm}^{2}$, length of the AML, short chordae, direct papillary muscle attachment, thickness of the basal segment of the interventricular septum and LV cavity size.

Valve models can be superimposed on CT images for accurate prediction of LVOT obstruction. In such cases, device alignment with the annular trajectory, adjustment of deployment height leading to variable lengths of the ventricular protrusion and intentional flaring of the ventricular aspect of the TMVR device should be considered (Figure 7). CT can identify device thrombosis or

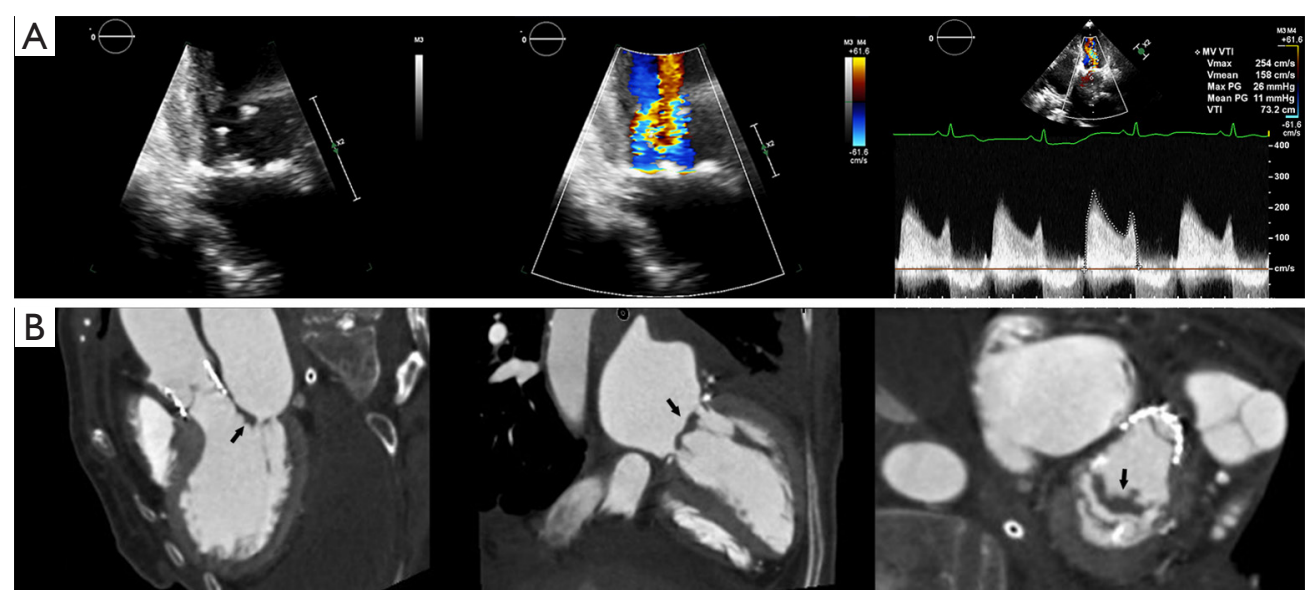

Figure 5 Severe, fibrotic, degenerative MS. (A) TTE: valve area: $1.3 \mathrm{~cm}^{2}$, mean gradient: $11 \mathrm{mmHg}$; HR: $76 \mathrm{bpm}$; (B) CT: fibrotic changes of AML (arrows), mild MAC. Note: (I) severity of MS does not corelate with the extent of MAC, or leaflet calcification, (II) TTE indicated leaflet calcification, not confirmed on CT. Degenerative MS is not uncommon in aortic stenosis patients; in this case, only the persistence of dyspnea after TAVR led to a more careful evaluation of mitral valve. TTE, transthoracic echocardiography; TAVR, transcatheter aortic valve replacement. 

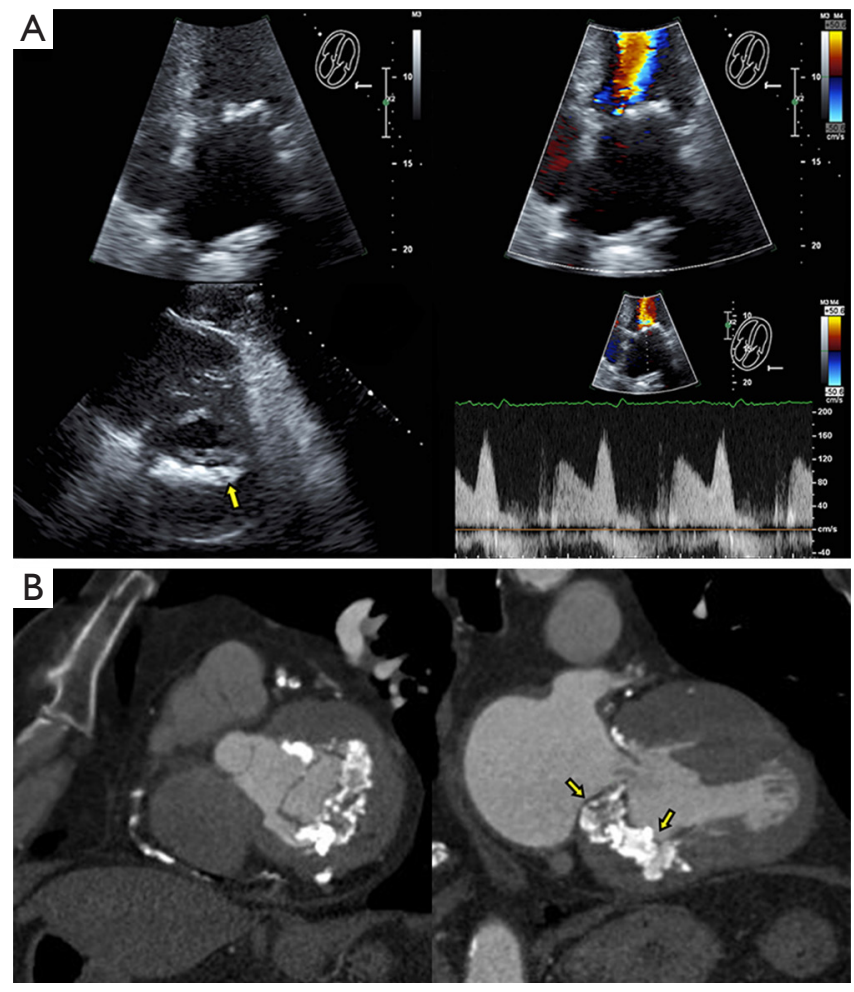

pannus, based on their different attenuation characteristics (Figure 8).

\section{Mitral valve technologies-imaging challenges and practical use}

Mitral valve interventions target the mitral leaflets, annulus and chords, or address post-procedure complications. They are divided in five respective categories: edge to edge repair, annuloplasty, chordal implants, TMVR and paravalvular leaks (PVL) repair.

(I) Edge to edge repair. MitraClip ${ }^{\circledR}$ technology replicates the Alfieri edge-to-edge stitch repair of MR and is exclusively driven by echocardiography (Figure 9 , Video 3$)$. It has been used in $>50,000$ patients, with FDA approval limited to DMR as a class IIb indication in patients with prohibitive surgical risk. It achieves MR reduction and may preclude surgical repair in failed cases (5). The echocardiographic patient selection criteria and the intraprocedural guidance principles have been extensively described $(5,27,28)$. Experienced operators commonly obtain patient, procedural and technical success, with acceptable device success, as defined by MVARC (9). The long-term hemodynamic impact of MitraClip ${ }^{\circledR}$ implantation has been less enthusiastically studied. Echocardiography-based mitral valve sizing and regurgitation quantification relies on several assumptions: single, static, rounded regurgitant orifice and single, central, non-eccentric, holosystolic jet, without associated MS. Post-clip deployment, these assumptions are violated, as a double orifice is created, frequently accompanied by two regurgitant jets. Area measurement of the double orifice is limited to the planimetry of cross-sectional views. A definition of MS post MitraClip ${ }^{\circledR}$ patients does not exist; a pressure halftime of $>91 \mathrm{~m} / \mathrm{sec}$ was found to correlate with high post-procedural gradients. Post-procedural mitral gradients are difficult to predict. Typically, they are mildly elevated compared to baseline and tend to not increase proportional to the number of clips implanted, even at higher heart rates, but raise significantly with exercise echocardiography. The residual regurgitation evaluation is limited to semi-quantitative color Doppler data, as PISA method is not validated for double orifices and jets. The impact of a persistent

Figure 7 TMVR—device landing zone planning: stereolithographic valve model, overlapped on 3DCT volumetric rendering. TMVR, transcutaneous mitral valve replacement. 


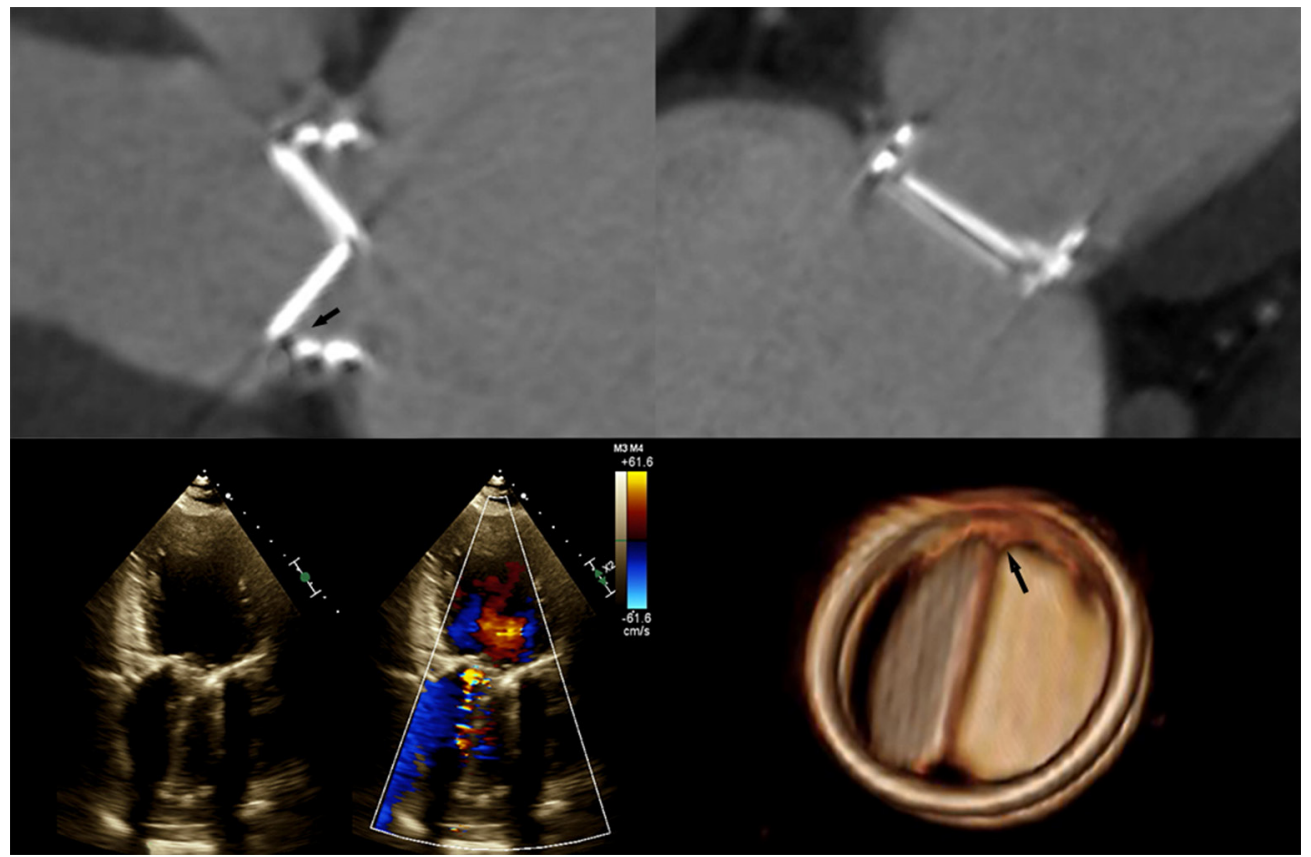

Figure 8 Mechanical mitral prosthesis: Patient referred for PVL evaluation, based on TTE (bottom left). CT 40\% systolic phase showed mild pannus (165 Hounsfield units) formation (bottom left), with incomplete systolic closure of the posterior disc (arrow) (top left), resulting in mild mitral regurgitation (arrow) (bottom right). No intervention was necessary. PVL, paravalvular leak; TTE, transthoracic echocardiography.

interatrial defect and shunt is not well understood and closure is advocated in patients with pulmonary hypertension and failing right ventricles. Notably, recent data shows absence of post implant LV remodeling in patients with $\mathrm{LVEF}<40 \%$, in contrast with earlier reports (29). Imaging MitraClip ${ }^{\circledR}$ patients remains largely operator-dependent requires development of specific criteria to clearly define device success and complications.

(II) Mitral annuloplasty. Largest experience stems from two TEE guided devices: Carillon ${ }^{\circledR}$ Mitral Contour System ${ }^{\circledR}$ and Cardioband ${ }^{\circledR}$ Mitral Valve Reconstruction System $(30,31)$. Carillon ${ }^{\circledR}$ is deployed into the coronary sinus via transjugular approach, then the device is anchored near the antero-lateral commissure, followed by plication of the peri-annular tissue, without general anesthesia, in FMR patients. A favorable anatomy of the coronary sinus in relation with the mitral annulus and left circumflex artery is a prerequisite for success (13). Cardioband ${ }^{\circledR}$ is delivered transseptally, reducing the transverse diameter of the mitral annulus by deploying multiple anchors along its posterior aspect, followed by adjustments of the device size, in FMR patients. Heavy mitral annulus or leaflet calcification are major exclusion criteria for Cardioband ${ }^{\circledR}$. Early reports suggest a steep learning curve, with compromise of left circumflex coronary artery for Carillon ${ }^{\circledR}$, or atrioventricular block and implant dehiscence resulting from anchor disengagement for Cardioband ${ }^{\circledR}$.

(III) Chordal implants. Initial data on the TEE-guided transapical Harpoon Mitral Valve Repair System is available (32). In DMR patients, artificial chords implantation reduces the annulus antero-posterior diameter and the mitral valve area.

(IV) TMVR relies on both echocardiography and CT, which are critical for patient selection, complete anatomic definition of the mitral complex, prediction of LVOT obstruction and complications diagnosis. Echocardiography is essential for procedural guidance $(18,19,21,24,26,33-35)$. The transseptal puncture site is chosen to allow positioning of the device coaxial with the MA trajectory. A landing zone is chosen under fluoroscopic and echocardiographic guidance, 

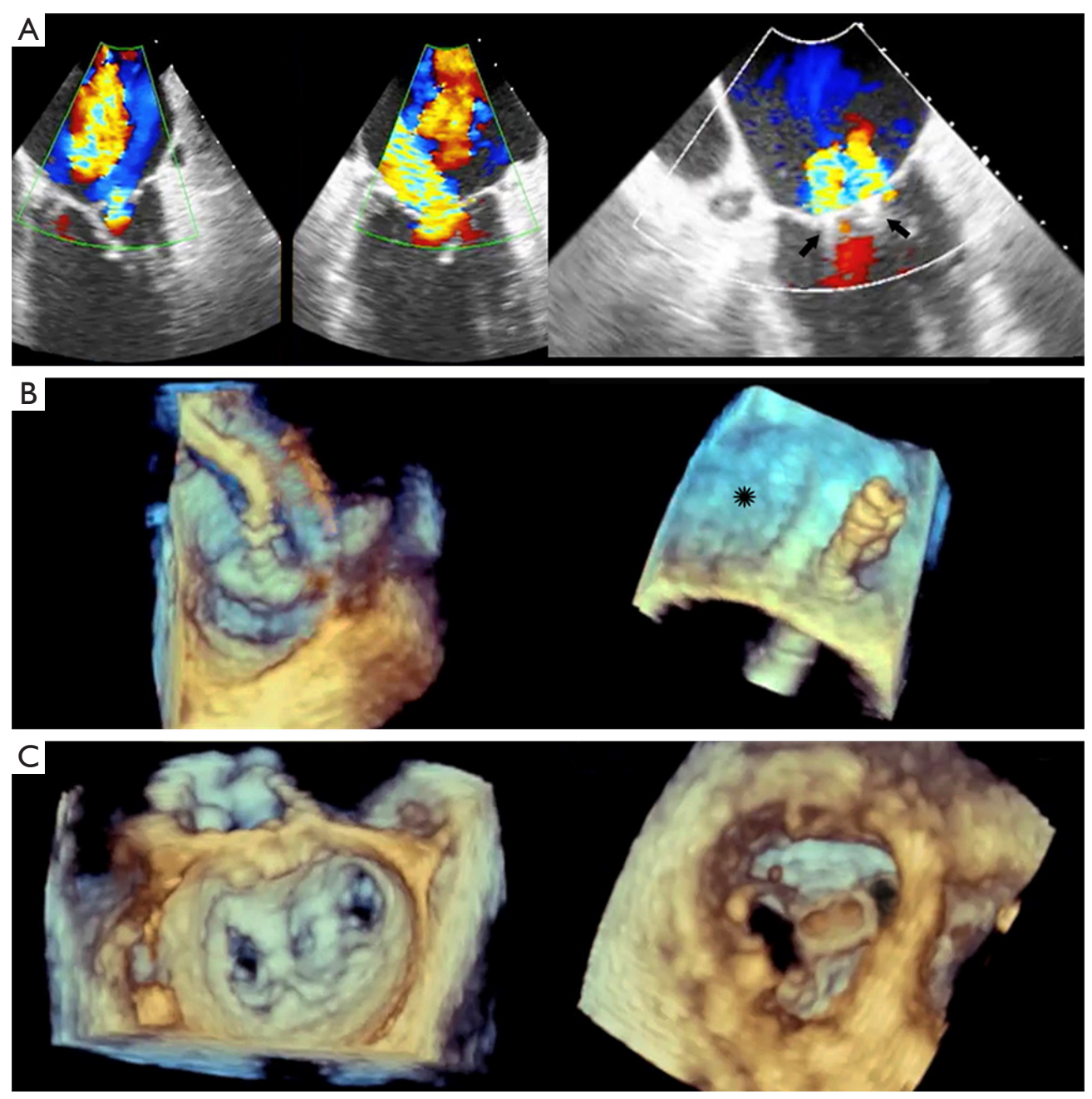

Figure 9 MitraClip $^{\circledR}$. (A) 2D TEE color: severe DMR (left quadrant); residual regurgitation post deployment of two clips (arrows) (right quadrant); (B) procedural guidance—clip crossing mitral valve (left quadrant), guide catheter across interatrial septum, missing fossa ovalis $\left({ }^{*}\right.$ ) (right quadrant); (C) 3D TEE: atrial view (left quadrant), ventricular view (right quadrant). TEE, transesophageal echocardiography.

monitoring for evidence of LVOT obstruction. 3D TEE is used to document full deployment of the atrial skirt and color Doppler helps detect perivalvular leaks. Sequential 2D X-plane views ascertain full leaflet capture by the device anchors, tabs or paddles, as TMVR devices do not rely on radial force for fixation. For apical delivery of TMVR devices, the location of left ventricular apical puncture is located by real time echocardiography as the operator "pokes" the ventricular apex, to identify a delivery trajectory coaxial with the MA trajectory (Figure 1) (14). TMVR procedures are divided in three categories: valve-in-valve, valve-in-ring, and valve-in-MAC
(Figures 10-12, Video 4).

(V) The Paravalvular Leak Academic Research Consortium expert statement describes endpoint definitions and the role of imaging (36). For mitral paravalvular leaks (PVL), TEE overcomes the left atrial shadowing from the prosthesis seen in TTE and precisely identifies the PVL location and size, allowing planning of the procedure and adequate communication with the operator. CT may identify prosthetic annulus dehiscence, incomplete sealing after TMVR, or early dysfunction of the prosthesis due to pannus, sometimes mimicking a PVL (Figure 8). CT imaging uses careful reconstructions, selected image kernels and multiphase analysis 

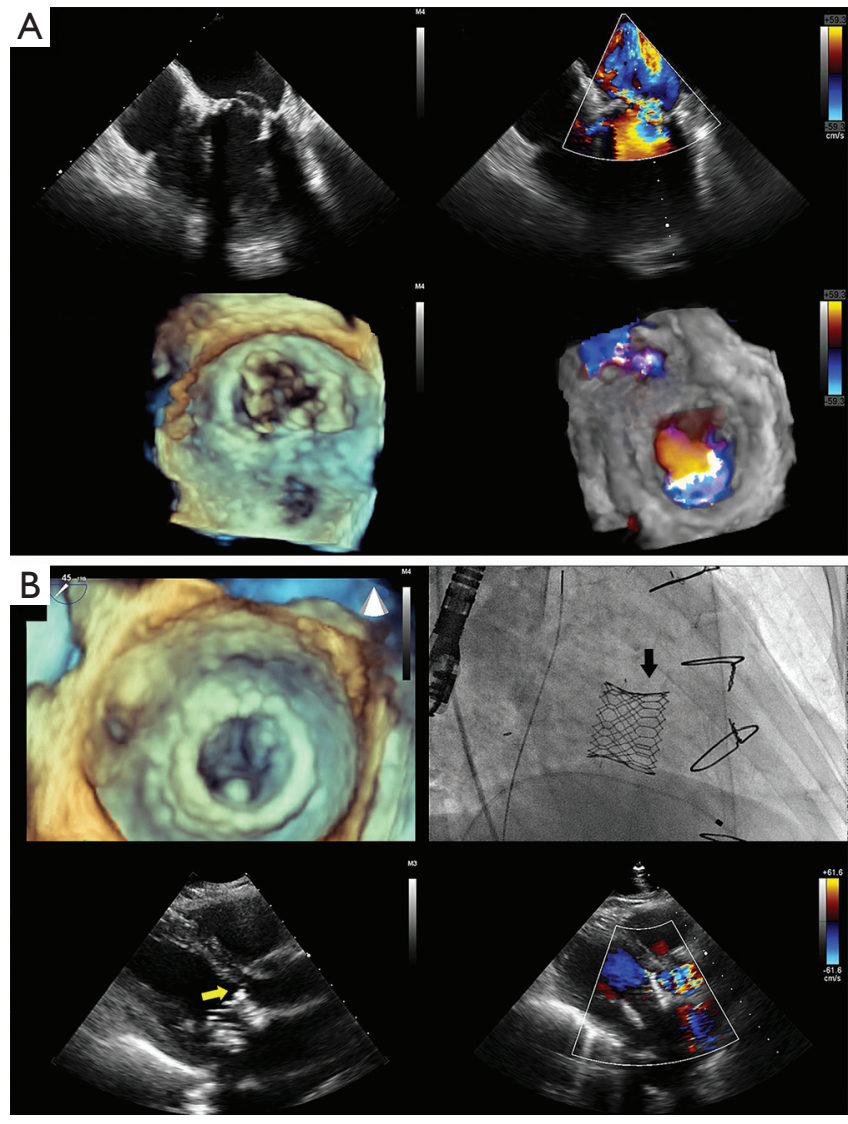

Figure 10 TMVR — valve in valve. (A) Biologic MV prosthesis, with flail leaflet, and severe MR. On color Doppler, note spontaneous PISA at Nyquist limit: $59 \mathrm{~cm} / \mathrm{sec}$; (B) post TMVR. Fluoroscopy shows flaring of the ventricular aspect of the prosthesis (black arrow). 2D TTE showed systolic LVOT obstruction (yellow arrow). Mean mitral gradient post-TMVR: $6 \mathrm{mmHg}$; HR: $67 \mathrm{bpm}$. TMVR, transcutaneous mitral valve replacement; TTE, transthoracic echocardiography; LVOT, left ventricular outflow tract.

to evaluate the anatomy of the leak and the surrounding structures. A PVL may be serpiginous and be located under, rather than adjacent to, the prosthetic ring, requiring dedicated oblique views for accurate imaging. Additionally, their area is dynamic and may seal during various phases of the cardiac cycle (Figure 13, Video 5). This phenomenon impacts the evaluation of the severity of mitral regurgitation, as the regurgitant jet may not be holosystolic and PISA may overestimate its severity. Additionally, the extent of associated hemolysis may be out of proportion to the size of the regurgitant jet, as estimated by color Doppler. In patients with
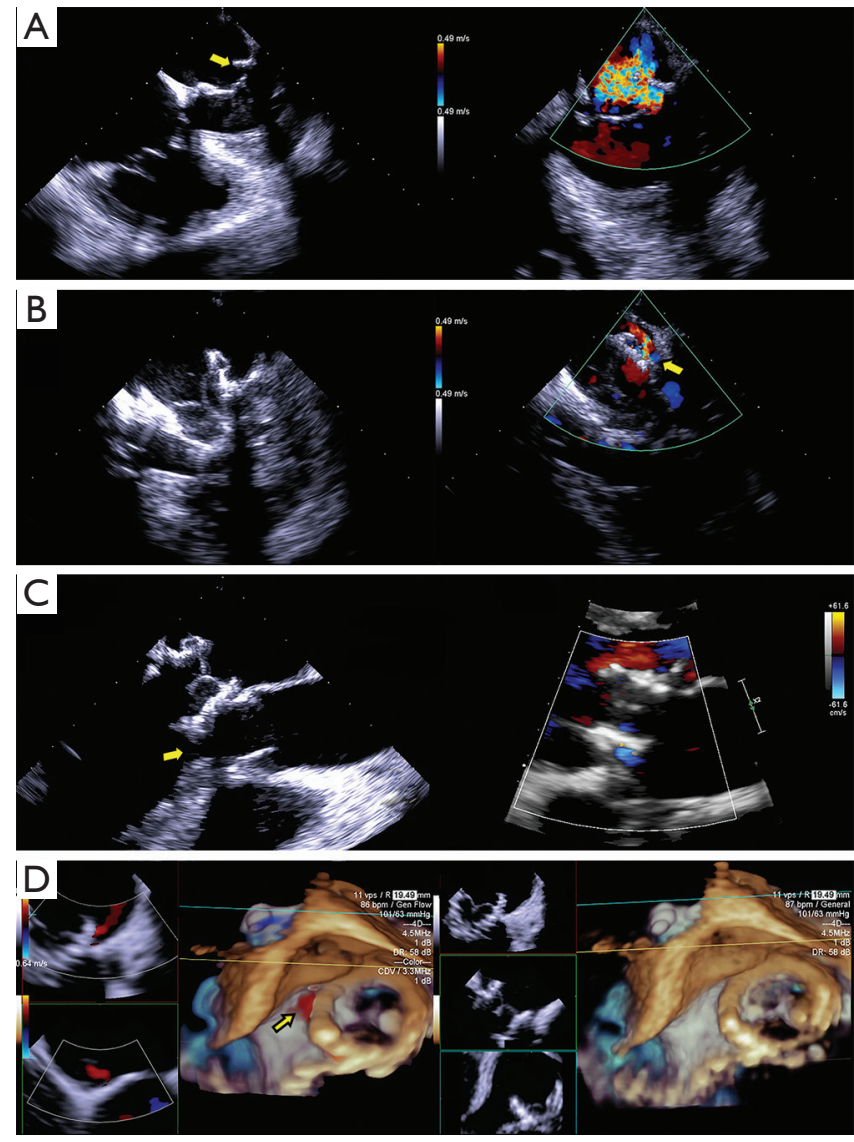

Figure 11 TMVR—valve in ring. (A) 3D TEE: severe MR post annuloplasty ring operation; (B) small PVL post TMVR. (C) 2D TEE LVOT laminar flow, without obstruction (arrow); (D) 3D TEE small, anterolateral PVL (arrow). Mean mitral gradient: pre-TMVR: $4 \mathrm{mmHg}$, post-TMVR: $7 \mathrm{mmHg}$; HR: 84-86 bpm. TMVR, transcutaneous mitral valve replacement; TEE, transesophageal echocardiography; PVL, paravalvular leak; LVOT, left ventricular outflow tract.

mechanical prostheses, TEE may predict the risk of disk impingement by the closure device and is used to determine the size of the closure device. Guiding the procedure, cropped 3D TEE and 2D X-plane views are used to ascertain the passage of the wire through the PVL orifice. Once the device is deployed, an immediate assessment of mechanical leaflet mobility is performed, followed by a search for residual leaks, frequently under hemodynamic stress (volume challenge and pharmacological manipulation of the arterial pressure). While PVL closure traditionally relies on a transseptal approach, a transapical "Hopscotch" approach has 

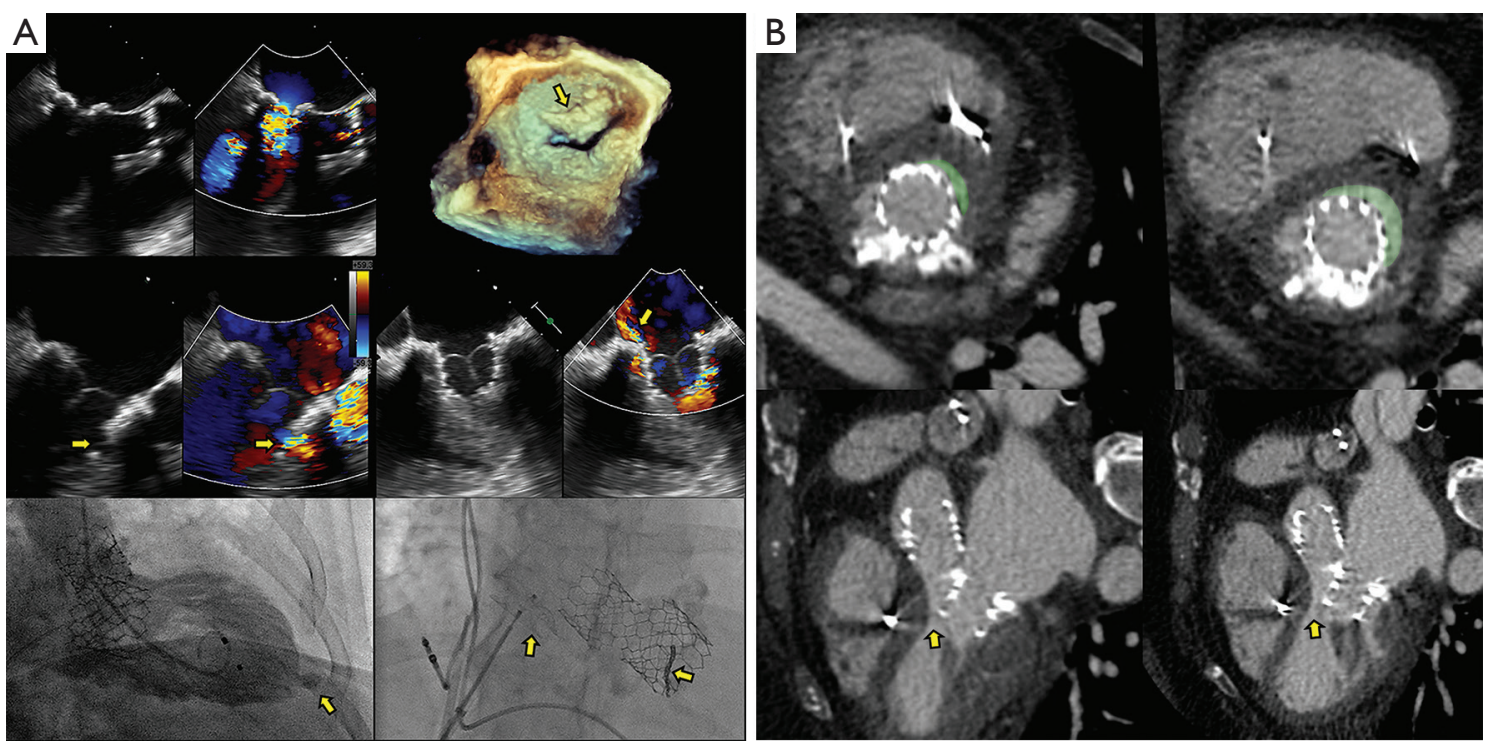

Figure 12 TMVR—valve in MAC. Concomitant TAVR and TMVR, in a patient with MAC, MS, and AS. Significant medial PVL (arrow), complete AV block, and severe LVOT obstruction resulted (arrow), with hemodynamic collapse. Mean mitral gradient: pre-TMVR: $6 \mathrm{mmHg}$, post-TMVR: $13 \mathrm{mmHg}$; HR: 71-66 bpm. Patient recovered after urgent septal perforator coiling, closure of the interatrial septum shunt with an Amplatzer ${ }^{\circledR}$ device (arrow), and permanent pacemaker implantation. Left ventricular perforation noted on LV angiogram (arrow), without pericardial tamponade. Patient with favorable clinical evolution, after prolonged hospitalization. (A) 2D and 3D TEE pre TMVR (top row), post TMVR (middle row) and fluoroscopic views (bottom row), showing LV apical perforation, Sapien ${ }^{\circledR}$ prostheses in mitral and aortic positions and septal perforator coils (arrow); (B) CT cross sectional LVOT view-semilunar green area: 40\% phase: $0.8 \mathrm{~cm}^{2}$ (left quadrant), $70 \%$ phase: $1.5 \mathrm{~cm}^{2}$ (right quadrant); note that the longitudinal LVOT view (bottom row) does not show a significant difference in LVOT diameter between the two phases (arrows). TMVR, transcutaneous mitral valve replacement; TAVR, transcatheter aortic valve replacement; PVL, paravalvular leak; TEE, transesophageal echocardiography; LVOT, left ventricular outflow tract.

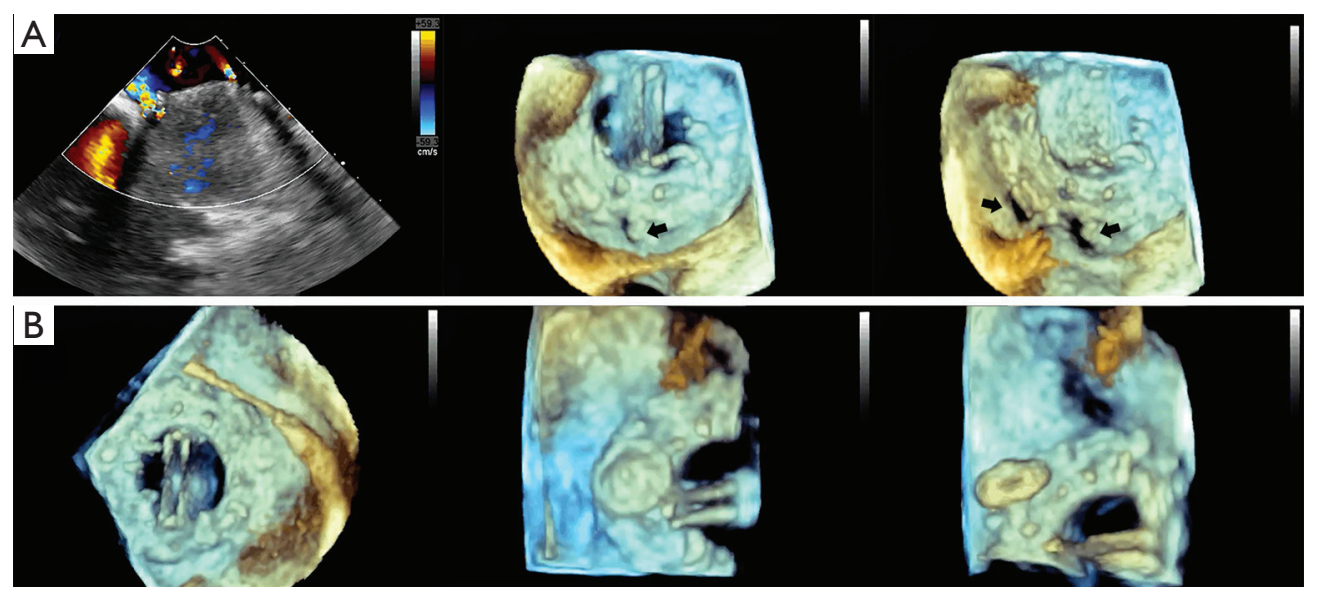

Figure 13 PVL. (A) Mechanical prosthesis with two distinct PVL jets; the main dehiscence (arrows) area is nearly completely shut in diastole (leaflets open) and clearly visible only in systole (leaflets closed); (B) guide catheter across interatrial septum, with wire protruding in the left atrium (left quadrant); atrial view of Amplatzer ${ }^{\circledR}$ device deployed over the medial aspect of the dehiscence (mid quadrant); ventricular view showing device incomplete apposition against the prosthetic ring (right quadrant). PVL, paravalvular leak. 
been described recently, which also benefits from echocardiographic intraprocedural guidance in combination with CT angiography (37).

\section{Mitral complex imaging-the future}

Patient-specific 3D printed cardiac models using stereolithographic reconstructions have been described.
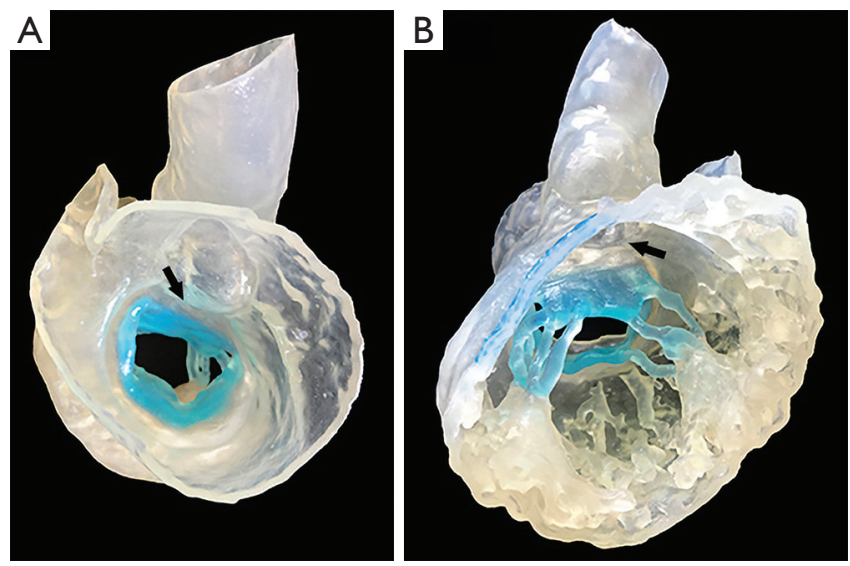

Figure 14 Hybrid 3D printing: 3D datasets fusion-3D TEE dataset used for segmentation of leaflets and chordae, CT dataset used for segmentation of papillary muscles, left ventricle, and aorta. (A) "Surgeon's view", including left atrial appendage (LAA), aorto-mitral curtain; (B) ventricular view, including LVOT. TEE, transesophageal echocardiography; LVOT, left ventricular outflow tract. Arrow, aorto-mitral curtain.
Commonly, either CT or 3D TEE datasets have been used for segmentation of cardiac structures. Hybrid imaging, using both CT and 3D TEE datasets for each patient, yields significantly more anatomic detail (38). Hybrid models take advantage of the strengths of each imaging modality and may benefit in the future from tissue-specific printing materials, which replicate the mechanical behavior of natural structures (Figure 14). Computational analysis of semi-automated 4D segmentations, specific to the mitral-aortic complex, may be used instead of prohibitively expensive printed models (Figure 15, Video 6). Singlescreen display of fused fluoroscopic and 3D TEE images facilitate the spatial location of various cardiac structures, possibly limiting procedure time and radiation exposure $(30,39)$. Fluid-structure interactions (FSI) models have been created, based on 3D imaging datasets. Numerical analysis of non-conforming mesh FSI models may represent a better way to develop and understand the impact of new mitral technologies.

\section{Conclusions}

The diagnostic imaging cardiologist bears the responsibility of a detailed preprocedural evaluation, should participate in procedure planning and provide procedural guidance leading to radiation exposure up to six-fold higher compared to the interventional operator (40). He or she must be proficient in multimodality imaging and possess considerable experience and technical skills. With this level of involvement, imaging now plays and will retain

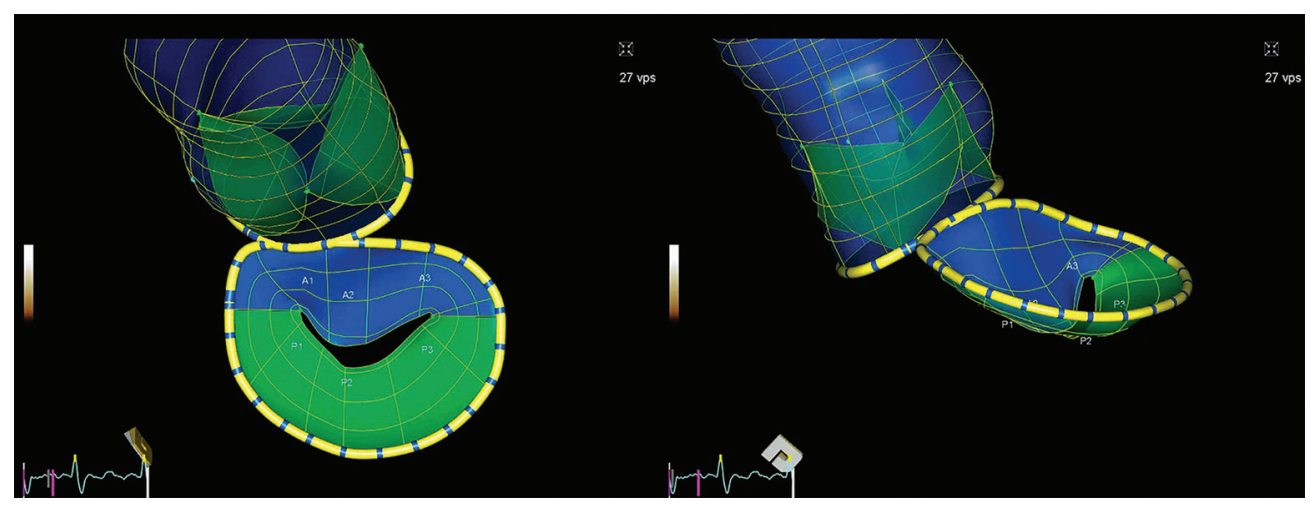

Figure 15 3D TEE valve model: bedside, real time segmentation, with automated analysis of anatomic landmarks. TEE, transesophageal echocardiography. 
in the future an unequivocally fundamental role in the development of mitral technologies.

\section{Acknowledgements}

The authors would like to thank Kevin Townsend from Materialise NV for his assistance in creating the 3D Printed Hybrid model.

\section{Footnote}

Conflicts of Interest: The authors have no conflicts of interest to declare.

\section{References}

1. Enriquez-Sarano M. Mitral Annular Disjunction: The Forgotten Component of Myxomatous Mitral Valve Disease. JACC Cardiovasc Imaging 2017;10:1434-6.

2. Eleid MF, Foley TA, Said SM, et al. Severe Mitral Annular Calcification: Multimodality Imaging for Therapeutic Strategies and Interventions. JACC Cardiovasc Imaging 2016;9:1318-37.

3. Wunderlich NC, Beigel R, Ho SY, et al. Imaging for Mitral Interventions: Methods and Efficacy. JACC Cardiovasc Imaging 2018;11:872-901.

4. Blanke P, Naoum C, Webb J, et al. Multimodality Imaging in the Context of Transcatheter Mitral Valve Replacement: Establishing Consensus Among Modalities and Disciplines. JACC Cardiovasc Imaging 2015;8:1191-208.

5. Katz WE, Conrad Smith AJ, Crock FW, et al. Echocardiographic evaluation and guidance for MitraClip procedure. Cardiovasc Diagn Ther 2017;7:616-32.

6. Naoum C, Leipsic J, Cheung A, et al. Mitral Annular Dimensions and Geometry in Patients With Functional Mitral Regurgitation and Mitral Valve Prolapse: Implications for Transcatheter Mitral Valve Implantation. JACC Cardiovasc Imaging 2016;9:269-80.

7. Marwick TH, Zoghbi WA, Narula J. Redrawing the borders: considering guideline revision in functional mitral regurgitation. JACC Cardiovasc Imaging 2014;7:333-5.

8. Izumo M, Akashi YJ. Exercise echocardiography for structural heart disease. J Echocardiogr 2016;14:21-9.

9. Stone GW, Adams DH, Abraham WT, et al. Clinical Trial Design Principles and Endpoint Definitions for Transcatheter Mitral Valve Repair and Replacement: Part 2: Endpoint Definitions: A Consensus Document From the Mitral Valve Academic Research Consortium. J Am Coll Cardiol 2015;66:308-21.

10. Kim JH, Kim EY, Jin GY, et al. A Review of the Use of Cardiac Computed Tomography for Evaluating the Mitral Valve before and after Mitral Valve Repair. Korean J Radiol 2017;18:773-85.

11. Wang DD, Eng M, Greenbaum A, et al. Predicting LVOT Obstruction After TMVR. JACC Cardiovasc Imaging 2016;9:1349-52.

12. Abdelghani M, Spitzer E, Soliman OII, et al. A simplified and reproducible method to size the mitral annulus: implications for transcatheter mitral valve replacement. Eur Heart J Cardiovasc Imaging 2017;18:697-706.

13. Blanke P, Dvir D, Naoum C, et al. Prediction of fluoroscopic angulation and coronary sinus location by CT in the context of transcatheter mitral valve implantation. J Cardiovasc Comput Tomogr 2015;9:183-92.

14. Blanke P, Park JK, Grayburn P, et al. Left ventricular access point determination for a coaxial approach to the mitral annular landing zone in transcatheter mitral valve replacement. J Cardiovasc Comput Tomogr 2017;11:281-7.

15. Kapadia SR, Mentias A, Barakat AF, et al. Relationship of mitral valve annulus plane and circumflex-right coronary artery plane: Implications for Transcatheter Mitral Valve Implantation. Catheter Cardiovasc Interv 2017;89:932-43.

16. Karady J, Ntalas I, Prendergast B, et al. Transcatheter mitral valve replacement in mitral annulus calcification "The art of computer simulation". J Cardiovasc Comput Tomogr 2018;12:153-7.

17. Korsholm K, Mortensen U, Jensen JM, et al. Transcatheter Mitral Paravalvular Leak Closure Facilitated by Preprocedural Cardiac CT for Simulation of Fluoroscopic Anatomy and Paravalvular Defect Localization. J Invasive Cardiol 2017;29:E23-5.

18. Mak GJ, Blanke P, Ong K, et al. Three-Dimensional Echocardiography Compared With Computed Tomography to Determine Mitral Annulus Size Before Transcatheter Mitral Valve Implantation. Circ Cardiovasc Imaging 2016;9.

19. Mooney J, Sellers SL, Ohana M, et al. Imaging for structural heart procedures: focus on computed tomography. EuroIntervention 2017;13:AA85-96.

20. Naoum C, Blanke P, Cavalcante JL, et al. Cardiac Computed Tomography and Magnetic Resonance Imaging in the Evaluation of Mitral and Tricuspid Valve Disease: Implications for Transcatheter Interventions. Circ Cardiovasc Imaging 2017;10.

21. Theriault-Lauzier P, Dorfmeister M, Mylotte D, et al. 
Quantitative multi-slice computed tomography assessment of the mitral valvular complex for transcatheter mitral valve interventions part 2: geometrical measurements in patients with functional mitral regurgitation. EuroIntervention 2016;12:e1021-30.

22. von Bardeleben RS, Colli A, Schulz E, et al. First in human transcatheter $\mathrm{COMBO}$ mitral valve repair with direct ring annuloplasty and neochord leaflet implantation to treat degenerative mitral regurgitation: feasibility of the simultaneous toolbox concept guided by 3D echo and computed tomography fusion imaging. Eur Heart J 2018;39:1314-5.

23. Blanke P, Dvir D, Cheung A, et al. A simplified D-shaped model of the mitral annulus to facilitate CT-based sizing before transcatheter mitral valve implantation. J Cardiovasc Comput Tomogr 2014;8:459-67.

24. Blanke P, Dvir D, Cheung A, et al. Mitral Annular Evaluation With CT in the Context of Transcatheter Mitral Valve Replacement. JACC Cardiovasc Imaging 2015;8:612-5.

25. Blanke P, Naoum C, Dvir D, et al. Predicting LVOT Obstruction in Transcatheter Mitral Valve Implantation: Concept of the Neo-LVOT. JACC Cardiovasc Imaging 2017;10:482-5.

26. Banks T, Razeghi O, Ntalas I, et al. Automated quantification of mitral valve geometry on multislice computed tomography in patients with dilated cardiomyopathy - Implications for transcatheter mitral valve replacement. J Cardiovasc Comput Tomogr 2018;12:329-37.

27. Hahn RT. Transcathether Valve Replacement and Valve Repair: Review of Procedures and Intraprocedural Echocardiographic Imaging. Circ Res 2016;119:341-56.

28. Wallenborn J, Herrmann S, Hansen M, et al. Systematic Echocardiographic Evaluation of Mitral Valve Regurgitation for Transcatheter Edge-to-Edge Repair. Echocardiography 2016;33:1069-79.

29. Schrage B, Kalbacher D, Schwarzl M, et al. Distinct Hemodynamic Changes After Interventional Mitral Valve Edge-to-Edge Repair in Different Phenotypes of Heart Failure: An Integrated Hemodynamic Analysis. J Am Heart Assoc 2018;7.

30. Pozzoli A, Zuber M, Taramasso M, et al. 3D echo-fluoro fusion imaging to guide Cardioband transcatheter mitral annuloplasty. Eur Heart J Cardiovasc Imaging 2018.

31. Mahmoud HM, Al-Ghamdi MA, Ghabashi AE. Real time three-dimensional transesophageal echocardiography guided coronary sinus cannulation during CARILLON mitral annuloplasty device therapy for a patient with chronic severe mitral regurgitation. Echocardiography 2015;32:181-3.

32. Gammie JS, Bartus K, Gackowski A, et al. BeatingHeart Mitral Valve Repair Using a Novel ePTFE Cordal Implantation Device: A Prospective Trial. J Am Coll Cardiol 2018;71:25-36.

33. Mackensen GB, Lee JC, Wang DD, et al. Role of Echocardiography in Transcatheter Mitral Valve Replacement in Native Mitral Valves and Mitral Rings. J Am Soc Echocardiogr 2018;31:475-90.

34. Thaden JJ, Malouf JF, Nkomo VT, et al. Mitral Valve Anatomic Predictors of Hemodynamic Success With Transcatheter Mitral Valve Repair. J Am Heart Assoc 2018;7.

35. Yoon SH, Whisenant BK, Bleiziffer S, et al. Transcatheter Mitral Valve Replacement for Degenerated Bioprosthetic Valves and Failed Annuloplasty Rings. J Am Coll Cardiol 2017;70:1121-31.

36. Ruiz CE, Hahn RT, Berrebi A, et al. Clinical Trial Principles and Endpoint Definitions for Paravalvular Leaks in Surgical Prosthesis: An Expert Statement. J Am Coll Cardiol 2017;69:2067-87.

37. Ruiz CE, Chi-Hion L, Vladimir J, et al. Hopscotch technique: A novel method for percutaneous closure of paravalvular leaks. Catheter Cardiovasc Interv 2017;89:944-50.

38. Kurup HK, Samuel BP, Vettukattil JJ. Hybrid 3D printing: a game-changer in personalized cardiac medicine? Expert Rev Cardiovasc Ther 2015;13:1281-4.

39. Faletra FF, Pozzoli A, Agricola E, et al. Echocardiographicfluoroscopic fusion imaging for transcatheter mitral valve repair guidance. Eur Heart J Cardiovasc Imaging 2018;19:715-26.

40. Salaun E, Carles S, Bigand E, et al. High Radiation Exposure of the Imaging Specialist During Structural Heart Interventions With Echocardiographic Guidance. JACC Cardiovasc Interv 2017;10:626-7.

Cite this article as: Loghin C, Loghin A. Role of imaging in novel mitral technologies-echocardiography and computed tomography. Ann Cardiothorac Surg 2018;7(6):799-811. doi 10.21037/acs.2018.09.07 